\title{
RedeR: R/Bioconductor package for representing modular structures, nested networks and multiple levels of hierarchical associations
}

\author{
Mauro AA Castro, Xin Wang, Michael NC Fletcher, Kerstin B Meyer and Florian Markowetz
}

\begin{abstract}
Visualization and analysis of molecular networks are both central to systems biology. However, there still exists a large technological gap between them, especially when assessing multiple network levels or hierarchies. Here we present RedeR, an R/Bioconductor package combined with a Java core engine for representing modular networks. The functionality of RedeR is demonstrated in two different scenarios: hierarchical and modular organization in gene co-expression networks and nested structures in time-course gene expression subnetworks. Our results demonstrate RedeR as a new framework to deal with the multiple network levels that are inherent to complex biological systems. RedeR is available from http://bioconductor.org/packages/release/bioc/html/RedeR.html.
\end{abstract}

\section{Rationale}

Biological networks contain modules of genes or proteins that may function in the same pathway [1]. As genes or proteins inside a module can be co-regulated, they are often represented by one single node in the network [2]. Such modules can be inferred by a number of statistical methods and the results are usually represented in graphs [3,4]. Given the complex associations that can take place in these graphs, it is a challenge to infer and visualize multiple levels or hierarchies within and between subnetwork structures.

Popular software like Cytoscape [5] provide a general framework to deal with part of this complexity by providing software plugins and visualizing networks in flat topologies. Flat networks are largely adequate to deal with different graph elements, as long as the network levels stay small. However, when describing and defining functional modules a hierarchical data structure is more appropriate because this enables the construction of graph elements within modules in a scalable system (for example, chains of nested networks). Herein we present RedeR, an R package combined with a Java core engine to cope with hierarchical and nested network structures.

\footnotetext{
* Correspondence: florian.markowetz@cancer.org.uk

Cancer Research UK Cambridge Research Institute and Department of Oncology, University of Cambridge, Robinson Way, Cambridge CB2 ORE, UK
}

RedeR is designed to deal with three key challenges in network analysis. Firstly, biological networks are modular and hierarchical, so network visualization needs to take advantage of such structural features to avoid cluttered and uninformative 'hairballs'. Secondly, network analysis relies on statistical methods, many of which are already available in resources like CRAN or Bioconductor. However, the missing link between advanced visualization and statistical computing makes it hard to take full advantage of $\mathrm{R}$ packages for network analysis. Thirdly, in larger networks user input is needed to focus the view of the network on the biologically relevant parts, rather than relying on an automatic layout function.

RedeR is designed to address these challenges: (i) we implement modular objects for subnetworks that allow to easily lay out and analyze network modules and their connections; (ii) the software is tightly integrated to $\mathrm{R}$ while RedeR visualizes $R$ outputs, its results can be directly fed back into $\mathrm{R}$ for further statistical analyses, which makes the power of $R$ available for users primarily interested in visualization but not statistical computing; and (iii) we implement a dynamic layout that directly reflects user input.

We exemplify RedeR's visualization and analysis capabilities in a case study based on the re-analysis of gene expression and chromatin immunoprecipitation (ChIP)on-chip data from an estrogen receptor (ER) study in 
the MCF-7 breast cancer cell line [6]. We anticipate that RedeR will be useful for integrative analyses and deriving gene expression networks that demand complex data abstraction and multiple network levels.

\section{Overview of the software}

RedeR is distributed as an R/Bioconductor package. It is implemented by S4 classes in R [7] combined with Java graphical user interface. Standard Java Swing components and the NetBeans IDE 6.9 development environment [8] were extensively used to implement the graphical interface, which operates in conjunction with $\mathrm{R}$ libraries. In what follows we describe the implementation of the main features of the software.

\section{User-friendly interface in $\mathbf{R}$}

The software uses different strategies to link $\mathrm{R}$ to Java (Figure 1). For the data interface, the callback engine makes calls from $R$ via xml-rpc protocol by setting $R$ as client and the Java app as server. For the graphic interface, the calls are executed from the Java core through dynamic libraries (that is, it wraps $\mathrm{R}$ graphics into Java classes). Four packages are essential to build the interface. At the Java side, the software uses the Apache xmlrpc webserver [9] and the JRI library interface [10]; at the $\mathrm{R}$ side, it uses the XMLRPC and rJava packages $[11,12]$. RedeR is invoked from $\mathrm{R}$ by the method 'calld':

\# Set the server port and invoke the Java app

$>$ library (RedeR)

$>$ rdp <- RedPort ( )

$>\operatorname{calld}(r d p)$

This method sets the environment and all paths required to start the callback engine, after which the software can either interact with $\mathrm{R}$ or run as a stand- alone application. The graphic interface is extensively controllable from the $\mathrm{R}$ command line and provides several menus that allow basic actions, such as selecting nodes and changing their appearance. In order to maintain a high level of compatibility, all methods in the $\mathrm{R}$ interface use igraph objects as prototype data format.

\section{Unique data structure for hierarchical networks}

The schematic representation of how data are stored in RedeR is depicted in Figure 2. The data structure emulates a mixed graph with two layers and multiple levels. The first layer can be defined by a directed acyclic graph (DAG) with no more than one parent for each vertex, essentially a data tree with multiple branches and levels, and with no cycles (Figure 2a). The second layer is designed as an undirected graph (UDG) on the lowest level of the DAG hierarchy. Horizontal or nonhierarchical associations can also be reassigned from one layer to another, external to the hierarchical structure. This data organization is depicted in Figure $2 \mathrm{~b}$ and corresponds to the topology that could represent a given dataset (for example, protein-protein interaction networks, ontologies, and so on). Flat networks, as illustrated in the left graph, can represent just one row instance of the data structure (that is, not divided into modules or layers). In contrast, hierarchical networks, as illustrated in the right graph, support a modular organization and can exhibit the complete information. The design of the software encapsulates the data structure within subclasses of a graph blueprint that contains all fields and methods common to both data types (Figure 2c). This design is implemented in the Java core and is extended to $\mathrm{R}$ to provide users and developers full access to the outer level of the application. As an initial demonstration of the software, next we provide a chunk

\section{statistical computing}

Network infe ence

Subnetwork identification

Time-course analysis

Clustering

Enrichment analysis

Differential networks

Topological analysis

...

* complete access to the Java app

* plugins coded in $\mathrm{R}$ and installed in Java

* extensible to standard R graph formats

\section{RedeR callback engine

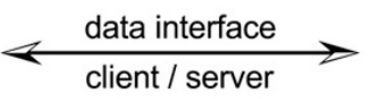 \\ \#Add graph objects >addGraph (port, g) \#Get graph objects $>$ g<-getGraph (port)}

graphic interface dynamic libraries

\section{nested networks}

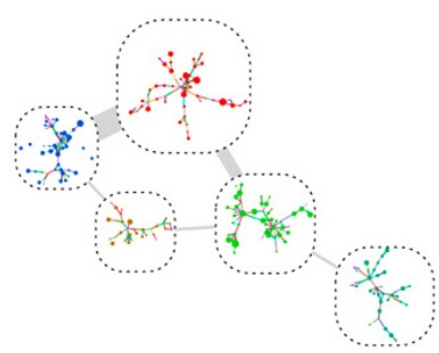

* sub-network representation and clustering * dynamic nested network layouts

* fully interactive

Figure 1 Schematic representation of the RedeR callback engine. In the low-level interface, the Apache xmlrpc webserver [9] is used to link $R$ and Java. 


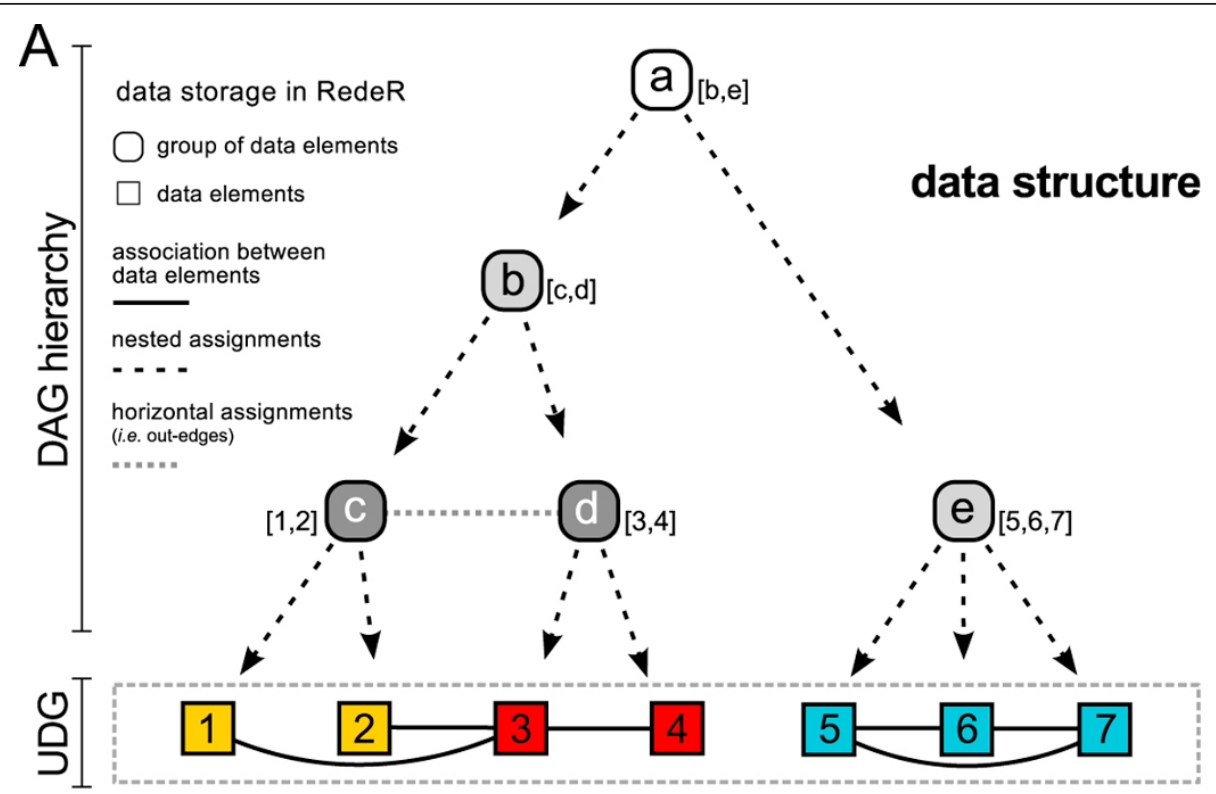

one row instance of the data structure

B

data abstraction
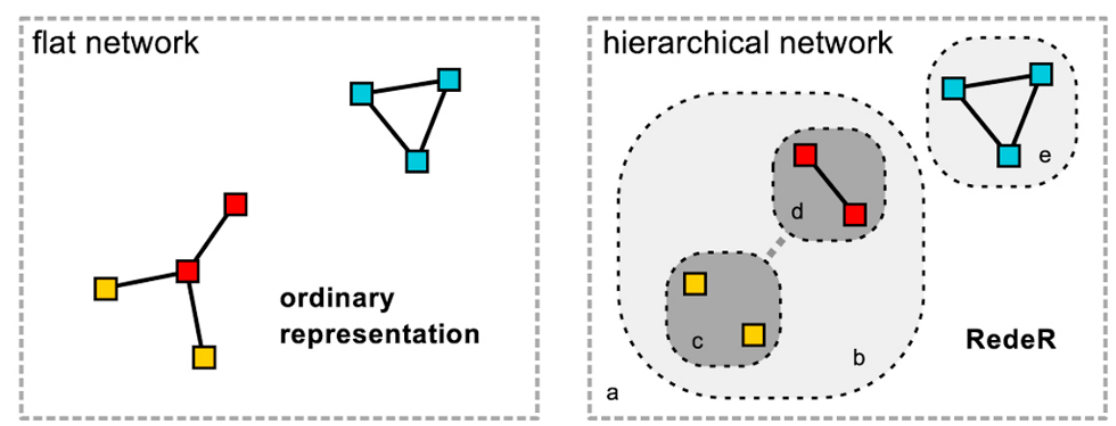

C data encapsulation and interface

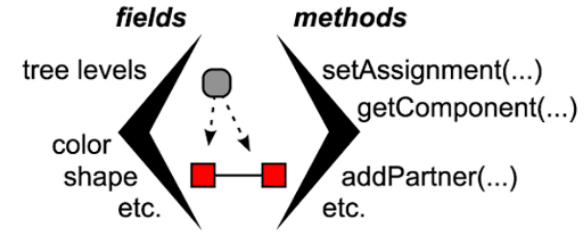

internal states

outside behaviors

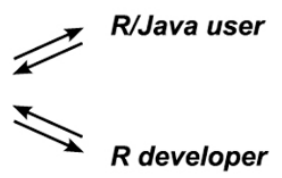

Figure 2 Schematic representation of RedeR data packing and storing. (a) Data structure. The software emulates a mixed graph with two layers and multiple levels in order to organize and manage the hierarchical associations. (b) Data abstraction. For the end-user, the data abstraction corresponds to the network layout that represents the data structure. A flat network is shown to contrast an ordinary representation (left) with RedeR hierarchical topology (right). (c) Data encapsulation: end-users and R developers have access to the outer level of the application through the methods handled by the interface.

of $\mathrm{R}$ that generates modular structures as illustrated in Figure $2 b$.

\# Generate an igraph object (a toy example with modular structures)

\footnotetext{
$>\mathrm{g}<-$ gtoy.rm $(\mathrm{m}=5, \mathrm{nmax}=30)$

\# Compute a hierarchical clustering using standard $R$ functions

$>$ hc<-hclust (dist (get.adjacency (g)) )
} 


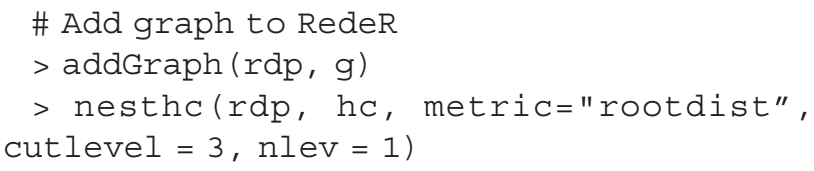

This toy example maps one level of the hierarchy onto the network topology. Additional levels and different sections of the hierarchy can be mapped using the same function (for further details, please see 'nesthc' documentation in the $\mathrm{R}$ package).

\section{Dynamic layout modeling}

One of the most versatile features of the software is the ability to deal with nested network objects using dynamic modeling, which makes it possible to represent, for example, subnetworks and time-series onto the same graph in a user-friendly routine. The layout uses forcedirected algorithms as described elsewhere [13,14]. Here we adapted the method to deal with nested networks. In force-directed graphs, each edge can be regarded as a spring - with a given target length - and can either exert a repulsive or attractive force on the connected nodes, while nodes are analogous to mutually repulsive charged particles that move according to the applied forces. In RedeR, the layout is additionally constrained by the hierarchical structure. For example, a nested node is constrained to its parent node by opposing forces applied by the nest, which is regarded as a special node whose nested objects can reach a local equilibrium independently from other network levels. The layout is adjusted by global options and evolves iteratively (and interactively) until the system reaches the equilibrium state. It can be started via either the graphical user interface or the $\mathrm{R}$ command line, as for example:

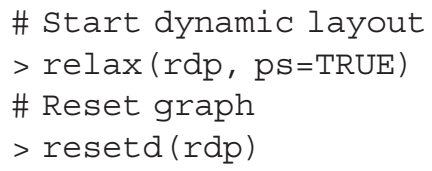

The user can observe all steps of the layout optimization process and, at any particular time, the process can be driven interactively. In this sense, 'dynamic' not only refers to the iteration steps required to layout a graph by the force-direct algorithm but also is related to the user's interaction. This option is particularly useful for additional control over containers and nested nodes in hierarchical structures. We also added to the Java core some popular static layout algorithms from open source libraries [15] as a complementary option to the list of all static layouts that can either be found in the $R$ package collections or, as usual, customized in $\mathrm{R}$ by the user.

\section{$R$ code deployment}

$\mathrm{R}$ developers can deploy $\mathrm{R}$ code to RedeR by using the 'PluginBuilder' method. This feature provides a direct way to extend existing $\mathrm{R}$ packages to the Java interface.
The combination of $\mathrm{R}$ and Java code in a mark-up construct gives rise to this extensible feature. The idea is based on the successful framework used by the Sweave package that mixes LaTeX syntax and R codes in order to parse $\mathrm{R}$ text chunks within LaTeX documents. In RedeR, the plugins are exported to the Java core by the 'submitPlugin' function. On the other side of the interface, the software receives the request, stores the new method in an XML document and mounts the plugin in the application, including submenus in the main panel. RedeR plugins have two main sections: methods and add-ons. The 'methods' section can be regarded as the plugin trigger. When installed in the Java app, this trigger starts a given analysis by unfolding $\mathrm{R}$ expressions wrapped in the methods. Add-ons use the same strategy, but remain hidden in the app and can either load formal functions or pass additional arguments to $\mathrm{R}$ (a code sample is provided in RedeR vignette, plugin builder tutorial).

\section{Pre-processed data and documentation}

The pre-processed data used in the case study were obtained by the LIMMA package [16]. An R script that reproduces the analysis is available in the supplements. Additionally, the $\mathrm{R}$ package provides extensive documentation for all methods available in the software, including description of the data objects, examples, and a tutorial introducing the main functionalities.

\section{Case study}

In this section we demonstrate some essential features of the software in two examples based on the re-analysis of ChIP-on-chip and gene expression data from a genome-wide study describing ER binding sites in the MCF-7 breast cancer cell line [6]. The ChIP-on-chip dataset consists of a Bed file containing the genome position of 3,665 unique ER binding sites, while the gene expression data consist of 12 time-course Affymetrix U133Plus2.0 microarrays from MCF-7 cells stimulated with estrogen for $0,3,6$ and $12 \mathrm{~h}$ (all arrays in triplicate).

\section{Biological background}

The purpose of the study by Carroll et al. [6] is the identification of new authentic cis ER binding sites and ER target genes in breast cancer cells. One of the challenges faced by the authors was that only a small fraction (4\%) of the ER binding sites mapped to promoterproximal regions, within $1 \mathrm{~kb}$ of the transcription start sites. More frequently, ER binding sites are found at considerable distance from the regulated gene and only one-third of early estrogen up-regulated genes contain ER binding sites within $50 \mathrm{~kb}$ of the transcription start site. This finding has made it difficult to validate ER- 
regulated candidate genes as there may be multiple genes within the $100 \mathrm{~kb}$ interval of the ER binding site and because the usual association of transcription factor binding sites and promoter regions occurs in only a minority of cases.

\section{Hierarchical and modular organization in gene co- expression networks}

The aim of this example is to examine the hierarchical structure of co-expressed gene network modules. A step-by-step description of the analysis is provided in Figure 3. Three standard $\mathrm{R}$ objects are used: a data frame object with many gene attributes inferred from the Carroll et al. [6] dataset (for example, differentially expressed genes, log fold change values and ER binding site distance), the corresponding gene expression matrix and an igraph object obtained by co-expression analysis on genes differentially expressed at $3 \mathrm{~h}$ (further details on the pre-processed data can be found in Additional file 1).
Figure 4a shows a hierarchical clustering obtained on the adjacency matrix and in Figure 4b we display two levels of such hierarchical organization onto the coexpression gene network. For each gene its size indicates the proximity to the nearest ER binding site (large nodes correspond to genes close to the ER binding sites) and the colors represent the log2 differential expression values.

Taken together, this case study not only illustrates how to constrain the network topology by a hierarchical structure, but also raises an interesting biological observation. The identification of co-regulated gene modules is one of the key steps towards understanding genetic regulatory networks. However, similar patterns in gene expression modules are not directly associated with a common mechanism of gene regulation. The identification of co-regulated modules is far from trivial and this case study provides a simple workflow to inspect in detail potentially co-expressed gene modules that share binding sites for the same transcription factor. The software permits visualizing these individual gene modules,

BOX 1: Hierarchical and modular organization in co-expressed gene networks.

\#---Step 1: load RedeR

library(RedeR)

rdp $<-$ RedPort()

calld(rdp)

\#--- Step 2: get a data frame and an igraph object (i.e. results from differential and co-expression analyses) data(ER.deg)

dt $<-$ ER.deg\$dat

sg <- ER.deg\$ceg

\#--- Step 3: map the data frame to the graph

$\mathrm{sg}<-$ att.mapv(sg, dat=dt, refcol=1)

\#--- Step 4: set attributes to RedeR (i.e. gene symbols, fold change and ER binding site distance to TSS)

$\mathrm{sg}<-$ att.setv(sg, from="Symbol", to="nodeAlias")

sg <- att.setv(sg, from="logFC.t3 ...t0", to="nodeColor", breaks=seq(-2,2,0.4), pal=2)

sg <- att.setv(sg, from="ERbdist", to="nodeSize", nquant=10, isrev=TRUE, $x \lim =c(5,40,1))$

\#--- Step 5: add graph to the app

addGraph(rdp,sg)

\#--- Step 6: compute a hierarchical clustering using standard $R$ functions on the adjacency matrix hc <- hclust(dist(get.adjacency(sg, attr="weight")))

\#--- Step 7: superimpose the dendrogram structure onto the network nesthc(rdp, hc, cutlevel $=3, \mathrm{nmemb}=5$, cex $=0.3$, labels $=\mathrm{V}(\mathrm{sg})$ \$nodeAlias)

\#--- Step 8: assign edges to containers

mergeOutEdges(rdp,nlev=2)

\#--- Step 9: relax the network and fine-tune layout interactively! relax(rdp)

\#--- Step 10: add color and size legends

addLegend.color(rdp, sg, title="diff. gene expression (logFC)")

addLegend.size(rdp, sg, title="bd site distance $(\mathrm{kb}) ")$

\#---Step 11: reset graph

resetd(rdp)

Figure 3 Hierarchical and modular organization in co-expressed gene networks. R script describing step-by-step all intermediate R objects required to obtain the results presented in the first case study. TSS, transcriptional start site. 


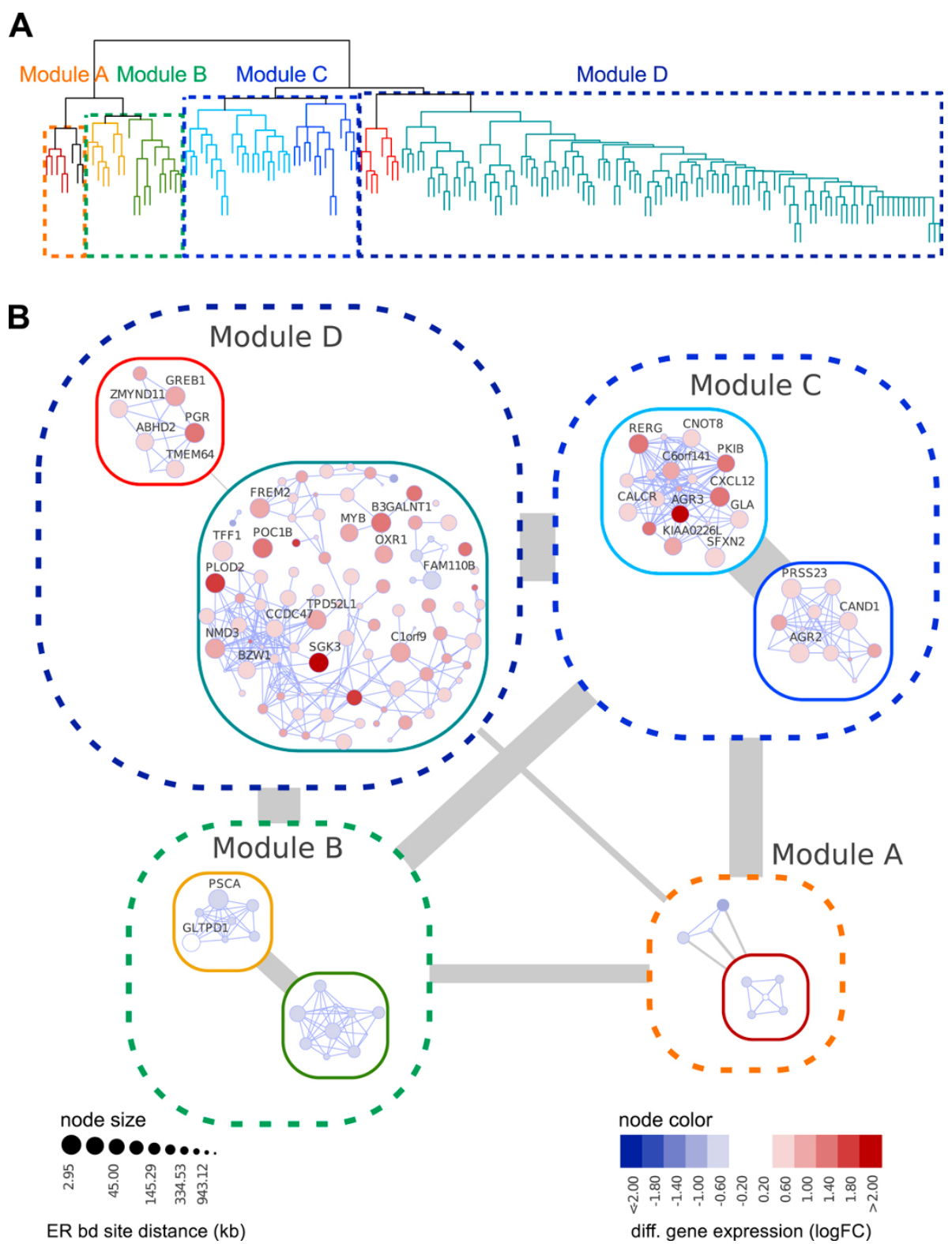

Figure 4 Hierarchical networks. (a) Dendrogram derived from complete-linkage clustering analysis using Euclidean distance on the gene expression matrix of all genes differently expressed at $3 \mathrm{~h}$ (related to $0 \mathrm{~h}$ ) in estrogen-treated MCF-7 cells (Carroll et al. [6] dataset). (b) Hierarchical network obtained by superimposing the dendrogram onto the corresponding co-expression gene network. The co-expression associations were computed for the same set of genes (for additional details on the pre-processed data please see Additional file 1 and Figure 3). Node coloring depicts differential expression as log2 fold-change (logFC) and node size indicates the kilobase distance of the transcription start site to the nearest ER binding site. Out-edge width represents the sum of all edges between modules divided by the total possible edges.

displaying each individual component and the connections between them, as well as the hierarchical associations between modules and genes.

\section{Nested structures in time-course gene expression subnetworks}

Another common approach to analyze complex biological datasets is the use of prior knowledge. Using the
HPRD database [17] as prior information, in Figure 5 we describe a workflow that maps to the human interactome all differentially expressed genes inferred in estrogen-treated MCF-7 cells (that is, genes differentially expressed at 3,6 and $12 \mathrm{~h}$ related to $0 \mathrm{~h}$ ), and for each time point we select the largest subnetwork in order to demonstrate how RedeR represents nested structures. 


\section{BOX 2: Nested structures in time-course gene expression subnetworks.}

\#--- Step 1: get a data frame and an interactome

data(ER.limma)

data(hs.inter)

dt $<-$ ER.limma

gi <- hs.inter

\#--- Step 2: extract a subgraph and set attributes to RedeR (i.e. $\log F C$ of t3-t0 contrast)

\#...p.s. not all genes are expected to be present in the interactome!

gt3 <- $\operatorname{subg}(g=g i$, dat=dt[dt\$degenes.t3! $=0$,$] , refcol=1)$

gt3 <- att.setv(g=gt3, from="Symbol", to="nodeAlias")

gt3 <- att.setv(g=gt3, from="logFC.t3...t0", to="nodeColor", breaks=seq(-2,2,0.4), pal=2)

\#--- Step 3: extract another subgraph and set attributes to RedeR (i.e. logFC of t6-t0 contrast)

gt6 <- subg $(g=g i$, dat=dt[dt\$degenes.t6!=0,], refcol=1)

gt6 <- att.setv(g=gt6, from="Symbol", to="nodeAlias")

gt6 <- att.setv(g=gt6, from="logFC.t6...t0", to="nodeColor", breaks=seq(-2,2,0.4), pal=2)

\#--- Step 4: extract another subgraph set attributes to RedeR (i.e. logFC of t12-t0 contrast)

gt12 <- subg(g=gi, dat=dt[dt\$degenes.t12!=0,], refcol=1)

gt12 <- att.setv(g=gt12, from="Symbol", to="nodeAlias")

gt12 <- att.setv(g=gt12, from="logFC.t12...t0", to="nodeColor", breaks=seq(-2,2,0.4), pal=2)

\#--- Step 5: add subgraphs to the app

addGraph(rdp, gt3, gcoord=c(10,25), gscale=20, isNest=TRUE, theme='tm1', zoom=30)

addGraph ( $r d p, g t 6$, gcoord $=c(20,70)$, gscale $=50$, isNest=TRUE, theme $='$ tm1', zoom=30)

addGraph $(\mathrm{rdp}, \mathrm{gt} 12, \mathrm{gcoord}=\mathrm{c}(70,55)$, gscale $=80$, isNest=TRUE, theme $=$ 'tm 1 ', zoom $=30$ )

\#--- Step 6: nest subgraphs (i.e. overlap time-series)

nestNodes(rdp, nodes=V(gt3)\$name, parent="N1", theme='tm2')

nestNodes(rdp, nodes=V(gt6)\$name, parent="N2", theme='tm2')

nestNodes $(\mathrm{rdp}$, nodes $=\mathrm{V}(\mathrm{gt} 3)$ \$name, parent="N4", theme='tm3')

\#--- Step 7: assign edges to containers

mergeOutEdges(rdp,nlev=2)

\#--- Step 8: relax the network

relax $(\operatorname{rdp}, 50,400)$

\#--- Step 9: add node colour legend (ps. same scale for all graphs)

$\mathrm{scl}<-$ gt 3 \$legNodeColor\$scale

leg <- gt3\$legNodeColor\$legend

addLegend.color(rdp, colvec=scl, labvec=leg, title="diff. gene expression (logFC)")

\#--- Step 10: select a gene

selectNodes(rdp,"RET")

\#--- Step 11: select and get nodes in a container

selectNodes(rdp,"N5")

sg <- getGraph(rdp, status="selected")

\#--- Step 12: reset graph

resetd(rdp)

Figure 5 Nested structures in time-course gene expression subnetworks. R script describing step-by-step all intermediate R objects required to obtain the results presented in the second case study. $\log F C, \log 2$ fold-change.

Accordingly, among the possible outcomes one may expect to see gene modules that are (i) induced early after stimulus, (ii) continuously stimulated/repressed, or (iii) respond later to the treatment. Given the partial- or non-overlapped time-course responses and the different module sizes, such a scenario can give rise to a complex data structure. Figure 6 shows these modules as a chain of nested subnetworks. Two observations are evident 

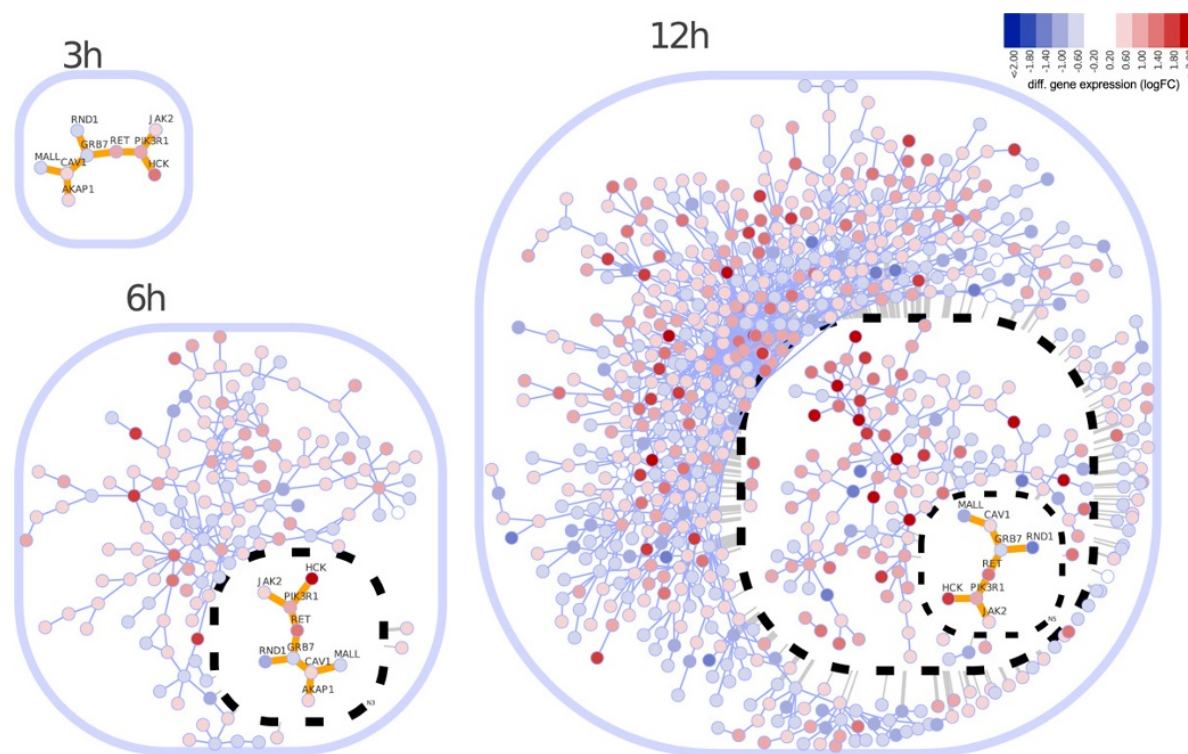

Figure 6 Nested subnetworks. Genes differentially expressed in estrogen-treated MCF-7 cells at 3, 6 or 12 h (relative to 0 h; Carroll et al. [6] dataset) were mapped to the human interactome (HPRD database [17]) and for each time point the largest subnetwork was selected in order to demonstrate how RedeR represents nested structures (additional details in Figure 5). Node coloring depicts differential expression as log2 fold-change (logFC). The insets correspond to the overlap between consecutive time points.

from this comparison across time series: as time goes by the subnetwork gets bigger but the core remains nearly the same. Such abstracted structure shows that the early response differentially expressed subnetworks are nested to the subsequent and larger gene modules, suggesting a stepwise and progressive gene expression response in estrogen-treated MCF-7 breast cancer cells. Additional file 1 provides supporting material to extend this case study to more advanced scenarios further illustrating the type of questions that can be explored by using RedeR.

Table 1 RedeR in the context of gold-standard network visualization software and $\mathbf{R}$

\begin{tabular}{|c|c|c|c|c|c|}
\hline & RedeR & Cytoscape & Graphviz & igraph & graph \\
\hline \multicolumn{6}{|l|}{ Software design } \\
\hline Hierarchical data structure ${ }^{a}$ & Yes $^{\mathbf{b}}$ & No & Yes $^{\mathbf{b}}$ & No & No \\
\hline Data abstraction ${ }^{a}$ & Modular ${ }^{\mathbf{b}}$ & Flat & Layered $^{\mathbf{b}}$ & Flat & Flat \\
\hline Data encapsulation ${ }^{a}$ & Yes & Yes & No & No & No \\
\hline Core engine & Java & Java & DOT & C & $\mathrm{R}$ \\
\hline $\mathrm{R}$ interface & $\mathrm{R}<->$ Java & R $->$ Java & $\mathrm{R}<-\mathrm{DOT}$ & $\mathrm{R}<-\mathrm{C}$ & Pure $R$ \\
\hline Deployment to $\mathrm{R}$ & Embedded & External & External & Embedded & Embedded \\
\hline Plugin coding language & $\mathrm{R}$ & Java & DOT language ${ }^{c}$ & $C^{d}$ & - \\
\hline \multicolumn{6}{|l|}{ Selected features } \\
\hline Scalability on nested networks ${ }^{e}$ & Yes & $\mathrm{No}^{f}$ & Yes & $\mathrm{No}^{\mathbf{g}}$ & $\mathrm{No}^{\mathbf{g}}$ \\
\hline Interactive graph handling & Yes & Yes & No & Partially ${ }^{\mathbf{h}}$ & No \\
\hline Dynamic layouts & Yes $^{\mathbf{i}}$ & No & No & No & No \\
\hline Comparison across multiple nested networks & Yes & No & No & No & No \\
\hline Panels $^{j}$ & Yes & No & No & No & No \\
\hline
\end{tabular}

${ }^{a}$ For further definitions, please see the Implementation section and Figure 2. ${ }^{b}$ In RedeR, the hierarchy is encapsulated, so users deal with methods. In Graphviz users implement methods by themselves in DOT language. This difference has an important effect on the ability of the software to deal with modular structures,

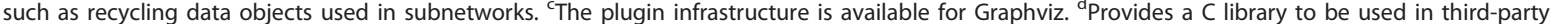
applications. ${ }^{e}$ Able to accommodate increasing amounts of nested objects. In Cytoscape, nested networks are represented as images inside nodes. For each nested network one image is required. Images are not scalable, so the hierarchy cannot be extended to other levels. ${ }^{9}$ In these applications, modules can be represented by layering images, which is essentially a drawing process. ${ }^{\mathrm{h}}$ According to the authors, the interactive features are not very well developed at this stage. IIn RedeR, the dynamic layout is also extensible to the nested structures. ${ }^{\text {J This }}$ feature provides support for multiple panels in the same graph, representing subnetworks, and so on. 


\section{Benchmark}

RedeR in the context of gold-standard software in the same field

There are other excellent tools to represent and analyze biological networks, and each has their own unique features. Table 1 describes RedeR in the context of gold standard software in the same field. In $R$ three packages provide standard software infrastructures to deal with graphs: igraph, graph and Rgraphviz [18-21]. However, the rendering process of these packages relies on R's internal plotting libraries, which lack interactive capabilities. Some simple interactive options are available in igraph, but according to the authors these features are not very well developed at this stage. In contrast, RedeR

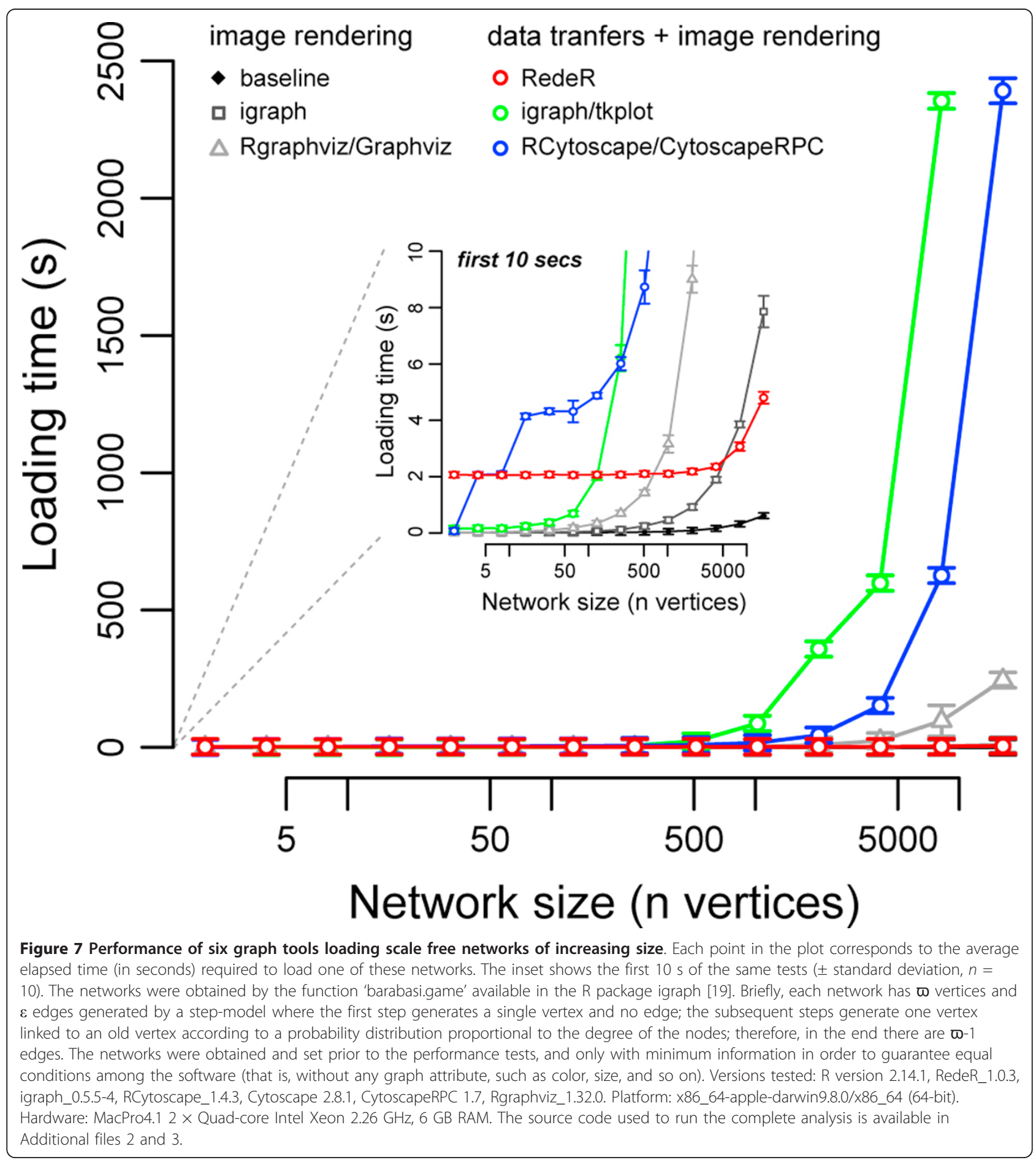


provides a robust graph-handling engine that is directly extensible to R objects; therefore, those used to work with graphs in R can easily become familiar with RedeR.

Another option is the package RCytoscape [22]. This $R$ package implements via CytoscapeRPC [23] an interface to Cytoscape [5], which can be regarded as a gold standard software for network visualization. Although robust and easy to use, Cytoscape is designed mainly to deal with flat network topology, which does not accommodate increasing amounts of nested objects. For example, using flat topology to represent a chain of nested networks, the number of graphs would increase proportionally with the network levels. Using RedeR, the job can be performed in just one graph (Figure $2 b$, data structure section). In this sense, RedeR constitutes a new option to assess networks with multiple levels or hierarchies, and this is a surprisingly common situation in biological networks.

\section{Performance}

In order to benchmark shared functionalities among these packages, we tested the performance for loading scale-free networks of increasing size, up to the human interactome scale. The results are shown in Figure 7 and the R script used to run the complete analysis is available in Additional files 2 and 3. Although any benchmark is restricted [24], it is clear from these results that RedeR performs very well, even compared to packages that only deal with image rendering. One remarkable aspect is that RedeR maintains its level of performance when tested by larger networks, only comparable to the baseline, which is a simple test to assess the speed of $\mathrm{R}$ for plotting dots and lines (that is, image rendering). The software continues to track the baseline, but transfers network information at the same time (Figure 7 , inset). Another remarkable aspect is observed at the maximum loading time. For example, while RedeR took $4.8 \mathrm{~s}( \pm 0.2 \mathrm{~s})$ to load a network with 16,384 nodes, RCytoscape/CytoscapeRPC required 2,391.3 s ( \pm $45.7 \mathrm{~s}$ ) to load the same graph (further details in the legend of Figure 7 and Additional files 2 and 3).

\section{Conclusions}

In this work we introduced RedeR, a software designed for the representation of nested and hierarchical biological networks. The ability to perform advanced visualization tightly integrated to $R$ allows Rede $R$ to take full advantage of $R$ packages for network analysis and statistical computing. Likewise, RedeR is an ongoing project that provides a comprehensive and entirely new framework to read, write and manipulate $\mathrm{R}$ code mixed to a Java data structure. Its architecture allows the creation of R-based plugins with minimum effort, potentially extending the existing $\mathrm{R}$ packages to different communities of users interested in studying biological networks.

Rather than analyzing a single network, current research focuses on differences in networks, re-wiring events, as well as higher-level, modular characteristics of networks. These can be hard to visualize in standard tools. RedeR implements a framework for network comparison and module representation by introducing a hierarchy of 'containers' in which many networks and their connections can be visualized at the same time. We anticipate that our software will be particularly useful to assess datasets that demand detailed analysis of inter- and intra-modular associations.

\section{System requirements}

$\mathrm{R}$ (version $>=2.14$ ) and Java Runtime Environment (version $>=5)$. Available since Bioconductor 2.9.

\section{Additional material}

Additional file 1: Pre-processed data analysis. PDF document describing the pre-processed data analysis, including three examples illustrating how RedeR can be integrated with other $R$ packages.

Additional file 2: Benchmark source code. R script used to run the benchmark analysis.

Additional file 3: Complementary benchmark source code. $R$ script with complementary $\mathrm{R}$ functions required in the benchmark.

\section{Abbreviations}

ChIP: chromatin immunoprecipitation; DAG: directed acyclic graph; ER: estrogen receptor.

\section{Acknowledgements}

We thank Professor Sir Bruce Ponder for his support. We also thank all FM lab members that kindly contributed with suggestions during the development of the R package. We acknowledge the support of The University of Cambridge, Cancer Research UK and Hutchison Whampoa Limited. The funders had no role in study design, data collection and analysis, decision to publish, or preparation of the manuscript.

\section{Authors' contributions}

Conceived the project: MAAC KBM FM. Supervised the project: KBM FM; Conceived and designed the software: MAAC XW FM. Implemented the software: MAAC. Implemented the analysis pipeline: MAAC XW. Conceived the case studies: MAAC XW MF KBM FM. Wrote the paper: MAAC KBM FM. All authors read and approved the final manuscript.

\section{Competing interests}

The authors declare that they have no competing interests.

Received: 6 February 2012 Revised: 23 March 2012

Accepted: 24 April 2012 Published: 24 April 2012

\section{References}

1. Luo F, Yang Y, Chen CF, Chang R, Zhou J, Scheuermann RH: Modular organization of protein interaction networks. Bioinformatics 2007, 23:207-214.

2. Han JD: Understanding biological functions through molecular networks. Cell Res 2008, 18:224-237.

3. Aittokallio T, Schwikowski B: Graph-based methods for analysing networks in cell biology. Brief Bioinform 2006, 7:243-255. 
4. Barabasi AL, Gulbahce N, Loscalzo J: Network medicine: a network-based approach to human disease. Nat Rev Genet 2011, 12:56-68.

5. Smoot ME, Ono K, Ruscheinski J, Wang PL, Ideker T: Cytoscape 2.8: new features for data integration and network visualization. Bioinformatics 2011, 27:431-432.

6. Carroll JS, Meyer CA, Song J, Li W, Geistlinger TR, Eeckhoute J, Brodsky AS, Keeton EK, Fertuck KC, Hall GF, Wang Q, Bekiranov S, Sementchenko V, Fox EA, Silver PA, Gingeras TR, Liu XS, Brown M: Genome-wide analysis of estrogen receptor binding sites. Nat Genet 2006, 38:1289-1297.

7. R Development Core Team: R: A Language and Environment for Statistical Computing. Vienna: R Foundation for Statistical Computing; 2011.

8. NetBeans IDE 6.9 Development Environment. [http://netbeans.org/].

9. Apache xmlrpc Webserver. [http://ws.apache.org/xmlrpc/].

10. JRI Library Interface. [http://www.rforge.net/JRI/].

11. Lang DT: XMLRPC: Remote Procedure Call (RPC) via XML in R.[http:// www.omegahat.org/XMLRPC/].

12. Urbanek S: rJava: Low-level R to Java interface.[http://www.rforge.net/ rJava/].

13. Brandes U: Drawing graphs: methods and models. In Lecture Notes in Computer Science. Volume 2025. Edited by: Kaufmann M, Wagner D. Heidelberg: Springer; 2001:71-86.

14. Fruchterman TMJ, Reingold EM: Graph drawing by force-directed placement. Software Practice Experience 1991, 21:1129-1164.

15. Java Universal Network/Graph Framework. [http://sourceforge.net/ projects/jung/].

16. Smyth GK: Linear models and empirical bayes methods for assessing differential expression in microarray experiments. Stat Appl Genet Mol Biol 2004, 3:Article3.

17. Prasad TS, Kandasamy K, Pandey A: Human Protein Reference Database and Human Proteinpedia as discovery tools for systems biology. Methods Mol Biol 2009, 577:67-79.

18. Le Meur N, Gentleman R: Analyzing biological data using R: methods for graphs and networks. Methods Mol Biol 2012, 804:343-373.

19. Csardi G, Nepusz T: The igraph software package for complex network research. $R$ package version 0.5.5-2 [http://cran.r-project.org/web/packages/ igraph/index.html]

20. Gentleman R, Whalen E, Huber W, Falcon S: graph: a package to handle graph data structures. $R$ package version 1.30 .30 [http://bioconductor.org/ packages/release/bioc/html/graph.html].

21. Gentry J, Long L, Gentleman R, Falcon S, Hahne F, Sarkar D, Hansen K: Rgraphviz: Provides plotting capabilities for $\mathrm{R}$ graph objects. $R$ package version 1.30.31 [http://bioconductor.org/packages/release/bioc/html/ Rgraphviz.html].

22. Shannon P: RCytoscape. $R$ package version 1.3 .0 [http://bioconductor.org/ packages/release/bioc/html/RCytoscape.html].

23. Bot JJ, Reinders MJ: CytoscapeRPC: a plugin to create, modify and query Cytoscape networks from scripting languages. Bioinformatics 2011, 27:2451-2452.

24. Norel R, Rice JJ, Stolovitzky G: The self-assessment trap: can we all be better than average? Mol Syst Biol 2011, 7:537.

doi:10.1186/gb-2012-13-4-r29

Cite this article as: Castro et al:: RedeR: R/Bioconductor package for representing modular structures, nested networks and multiple levels of hierarchical associations. Genome Biology 2012 13:R29.

\section{Submit your next manuscript to BioMed Central and take full advantage of:}

- Convenient online submission

- Thorough peer review

- No space constraints or color figure charges

- Immediate publication on acceptance

- Inclusion in PubMed, CAS, Scopus and Google Scholar

- Research which is freely available for redistribution

Submit your manuscript at www.biomedcentral.com/submit
Ciomed Central 